\title{
Synthesis of Some New Halogenated N-Thiazolyl Substituted Hydroxy Acid Amides and Their Use as Possible Fungicides ${ }^{\dagger}$
}

\author{
H. Tripathy, D. G. Pradhan, B. C. Dash and G. N. Mahapatra \\ Department of Chemistry, Ravenshaw College, Utkal University, Cuttack-3, India
}

Received November 8, 1972

\begin{abstract}
A large number of new N-thiazolyl, chloro-thiazolyl, brome-thiazolyl and iodothiazolyl amides of salicylic, hydroxy naphthoic acids, both halogenated and non-halogenated ones have been synthesised by condensing simple as well as halogenated thiazoles with different aromatic hydroxy acids, viz. salicylic, chloro- and bromo-salicylic, hydroxy naphthoic and bromo hydroxyl naphthoic acids. All these compounds are assayed for their fungicidal activity using Piricularia oryzae (Cav.), Helminthosporium oryzae and Carvularia lunata as the test fungi. An attempt has been made to establish a relationship between chemical structure and fungicidal activity.
\end{abstract}

The use of salicylic acid, salicylanilides and their halogen derivatives are quite well known for their fungicidal properties. Some of the halogen derivatives of salicylanilides are used in the treatment of epidermphytosis. ${ }^{11}$ Multifungin, a trade name for 5-bromo salicyl-4chloro anilide is a commercial fungicide used in the treatment of human fungus-diseases. Bromo salicylic acid shows marked fungicidal activity $^{2}$ against fungus-diseases in plant.

Hydroxy naphthoic acids and their anilides have also shown marked antifungal activity. ${ }^{3 \text { ) }}$ The use of thiazole derivatives including halogenated ones as fungicides has gained importance during the recent years."

In view of the above observations, it was considered worthwhile to synthesize a few anilides by condensing salicylic acid, halogenated salicylic acids, hydroxy naphthoic acids and bromo hydroxy naphthoic acids in one hand and thiazolyl amines and their chloro-, bromo-, and iododerivatives on the other hand, with the hope that the resulted anilides which contain all the potential fungicidal groupings, i.e. thiazole moiety, halogens and aromatic hydroxy acids in a single molecule would certainly show powerful fungicidal activity. Further, to compare the effectiveness of these

$\dagger$ Search for New Fungicides. Part I. hydroxy amido thiazoles some aromatic amines have also been condensed with the hydroxy acids with the view to have a comparative evaluation of the fungicidal activity of the thiazole moiety and to arrive at a conclusion regarding the relation between chemical structure and fungicidal activity of such compounds.

\section{EXPERIMENTAL PROCEDURE}

1. 3: 5-Dichloro-salicylic acid. Salicylic acid (5. $0 \mathrm{~g})$ was dissolved in minimum volume of glacial acetic acid and to this solution dry chlorine gas was bubbled for $2 \mathrm{hr}$ with occasional shaking when a white crystalline solid separated. The residue was collected by filtration, washed with excess of water and finally crystallized from ethanol; yield $80 \% ; \mathrm{mp} 219^{\circ} \mathrm{C}$ (Found: $\mathrm{Cl}, 34.58 ; \mathrm{C}_{7} \mathrm{H}_{4} \mathrm{O}_{3} \mathrm{Cl}_{2}$ requires $\mathrm{Cl}, 34.30 \%$ ).

2. 5-Bromo-salicylic acid. Salicylic acid $(7.0 \mathrm{~g})$ was dissolved in minimum volume of glacial acetic acid and was taken in a dry conical flask kept in icebath and was stirred mechanically. Bromine $(8.0 \mathrm{~g})$ in glacial acetic acid $(15 \mathrm{ml})$ was then added dropwise from a separating funnel. Stirring was continued for a further period of $30 \mathrm{~min}$ at room temperature and then the resulting solution was poured into large volume of water. A white precipitate was obtained which was collected by filtration, washed with water and finally crystallised from ethanol; yield $65 \%$; mp $157^{\circ} \mathrm{C}$ (Found: $\mathrm{Br}, 36.21 ; \mathrm{C}_{7} \mathrm{H}_{5} \mathrm{O}_{3} \mathrm{Br}$ requires $\mathrm{Br}, 36.86 \%$.

3. 4-Bromo-1-hydroxy-2-naphthoic acid. 1-Hydroxy-2-naphthoic acid $(9.5 \mathrm{~g})$ was brominated with 
bromine $(8.0 \mathrm{~g})$ in glacial acetic acid medium by the method outlined in 2. Yield $85 \% ; \operatorname{mp~} 238^{\circ} \mathrm{C}$ (Found: $\mathrm{Br}, 29.80 ; \mathrm{C}_{11} \mathrm{H}_{7} \mathrm{O}_{3} \mathrm{Br}$ requires $\mathrm{Br}, 30.00 \%$ ).

4. 4-Bromo-3-hydroxy-2-naphthoic acid. 3-Hydroxy-2-naphthoic acid $(9.5 \mathrm{~g})$ was brominated with bromine as above. Yield $80 \%$ mp $233 \sim 34 \mathrm{C}$, (Found: $\mathrm{Br}, 29.71 ; \mathrm{C}_{12} \mathrm{H}_{7} \mathrm{O}_{3} \mathrm{Br}$ requires $\mathrm{Br}, 30.00 \%$ ).

5. 2-Amino-4-phenyl-5-chloro-thiazole. 2-Amino4-phenyl thiazole $(2.0 \mathrm{~g})$ was treated with $\mathrm{HCl}$ (Conc. $3.0 \mathrm{ml}$ ) and the hydrochloride salt was dissolved in glacial acetic acid $(25 \mathrm{ml})$, and the mixture was stirred well. Dry chlorine gas was bubbled at a slow and steady rate through the above hydrochloride solution of the thiazole, with occasional shaking until a solid separated. The residue was separated by filtration and was treated with liquor $\mathrm{NH}_{4} \mathrm{OH}$ to liberate the free base. This was collected by filtration and finally crystallised from ethanol. Yield $68 \%$ mp $160^{\circ} \mathrm{C}$ (Found: $\mathrm{S}, 15.18 ; \mathrm{Cl}, 16.52 ; \mathrm{C}_{9} \mathrm{H}_{7} \mathrm{~N}_{2} \mathrm{SCl}$ requires $\mathrm{S}$, $15.20 ; \mathrm{Cl}, 16.86 \%$

6. 2-Amino-4-phenyl-5-bromo-thiazole. 2-Amino4-phenyl thiazole was brominated by the method of Mahapatra. ${ }^{5}$

7. 2-Amino-4-phenyl-5-iodo-thiazole. 2-Amino-4phenyl thiazole was iodinated by the method of Garreau $^{6}$ with certain modifications. 2-Amino-4phenyl thiazole $(2.0 \mathrm{~g})$ was treated with pure and redistilled $\mathrm{HI}(6.5 \mathrm{ml})$ and the hydroiodide salt was dissolved in glacial acetic acid $(25 \mathrm{ml})$ and the mixture was kept stirring mechanically on an ice-bath. Pure and resublimed iodine $(3 \mathrm{~g})$ in glacial acetic acid $(30 \mathrm{ml})$ was added dropwise. The stirring was continued for one more hour and the resulting solution was allowed to stand at room temperature for $15 \mathrm{~min}$ and then a pinch of red oxide of mercury was added and refluxed on a water-bath for $30 \mathrm{~min}$. The excess of the solvent was distilled off and the oily mass was poured into large excess of water. The gummy residue separated was treated with liquor $\mathrm{NH}_{4} \mathrm{OH}$ to liberate the free base. The product was separated by filtration and finally crystallised from ethanol. Yield $65 \%$; mp $103^{\circ} \mathrm{C}$ (Found: I, 40.28; $\mathrm{C}_{9} \mathrm{H}_{7} \mathrm{~N}_{2} \mathrm{SI}$ requires $\mathrm{I}$, $42.06 \%$ ).

8. 2-(a-Hydroxyl- $\beta$-naphthoylamido)-4-phenyl-thiazole. 1-Hydroxy-2-naphthoic acid $(1.0 \mathrm{~g})$ was taken in a clean and dry conical flask. To it was added thionyl chloride $(2 \mathrm{ml})$ and the resulting mixture was refluxed on a water-bath for about an hour so as to convert the acid completely into the acid chloride. Excess of thionyl chloride was driven off, the residue was cooled and to it was added a solution of 2-amino4-phenyl thiazole $(1.0 \mathrm{~g})$ in dry acetone. It was then refluxed on a water-bath for $10 \mathrm{~min}$ and then water $(50 \mathrm{ml})$ was added. The solid was collected by filtration, washed thoroughly and finally crystallised from ethanol. Yield $65 \%$; $\mathrm{mp} 200^{\circ} \mathrm{C}$ (Found: S, 9.62; $\mathrm{C}_{20} \mathrm{H}_{14} \mathrm{~N}_{2} \mathrm{O}_{2} \mathrm{~S}$ requires $\mathrm{S}, 9.25 \%$ ).

The condensation of halogenated hydroxy acids with non-halogenated and halogenated 2-amino thiazoles was carried out as above. The mp, yield and analytical data of such hydroxy acid amido compounds are given in table $\mathbf{I}$.

\section{Fungicidal test}

For the fungicidal assay of these compounds, the method of Montgomery and Moore, ${ }^{7}$ with slight modification was used. Piricularia oryzae (Cav.), Helminthosporium oryzae, the causative organisms of rice blast and Carvularia lunata, the fungus causing brown spots in rice plants have been used as the test fungi. Some of the compounds (numbered 8, 9, 17, $18,26,27,35,36,52,53,61,62,70,71,79$ and 80 in Table I) which show maximum fungicidal activity against Piricularia oryzae (Cav.) are also tested against Helminthosporium oryzae. The results as evident from Table I show that these are also very active against Helminthosporium oryzae. The chemicals in different concentrations were kept in contact with the spores for $24 \mathrm{hr}$. The percentage of germination of spores at $10 \mathrm{ppm}$ and $1 \mathrm{ppm}$ of the fungicides is given in Table I.

Analysis of the results of fungicidal activity shows a definite pattern from which the following conclusions can be derived:

i) The thiazole nucleus attached to the amide nitrogen atom definitely improved the fungicidal activity in comparison to the aromatic nucleus attached to the same nitrogen.

ii) Salicylamides are more active than the naphthoic acid amides.

iii) Introduction of halogen to the acid part imporved the fungicidal activity over the non-halogenated acid amides. Chloro derivatives are found to have greater fungicidal property than the bromo derivatives.

iv) When halogen is introduced into the thiazolyl part the fungicidal activity is augmented.

v) The most active compounds are those in which chlorine is on both the acid and thiazolyl parts, except in a few cases.

vi) The position of the hydroxyl group in the naphthoic acid either in $\alpha$ or $\beta$ position does not change the fungicidal activity in a conclusive way.

vii) These compounds are most active towards Piricularia oryzae (Cav.), less towards Helminthosporium oryzae and least towards Carvularia lunata. All these are disease-causing fungi of rice plant. 
Table I. Analytical Data and Fungicidal Results of N-Thiazolyl

Substituted Hydroxy ACID Amides

\begin{tabular}{|c|c|c|c|c|c|c|c|c|c|c|}
\hline \multirow[b]{2}{*}{$\begin{array}{l}\text { Sl. } \\
\text { No. }\end{array}$} & \multirow[b]{2}{*}{ Nature of $R$. } & \multirow[b]{2}{*}{$\operatorname{mp}_{{ }^{\circ} \mathrm{C}}$} & \multirow[b]{2}{*}{$\underset{\%}{\text { Yield }}$} & \multicolumn{2}{|c|}{ Halogen $(\%)$} & \multicolumn{2}{|c|}{ Sulphur $(\%)$} & \multicolumn{3}{|c|}{ Fungicidal results } \\
\hline & & & & Found & Calc. & Found & Calc. & $\begin{array}{c}\text { Ger. at } \\
10 \mathrm{ppm} \\
\%\end{array}$ & $\begin{array}{l}\text { Ger. at } \\
1 \mathrm{ppm} \\
\%\end{array}$ & Test fungus \\
\hline 1 & 2 & 3 & 4 & 5 & 6 & 7 & 8 & 9 & 10 & 11 \\
\hline
\end{tabular}

(Fungicidal assay in control-93 95\% germination)

1. $\beta$-Naphthyl

2. (o) Chlorophenyl

3. 4-Phenyl-2-thiazolyl

4. 4-( $\beta$-Naphthyl)2-thiazolyl

5. 4-( $a$-Naphthyl)2-thiazolyl

6. 4-(p-Hydroxyphenyl)2-thiazolyl

7. 4-( $p$-Methoxyphenyl)2-thiazolyl

8. 4-(p-Chlorophenyl)2-thiazolyl

9. 4-(p-Bromophenyl)2-thiazolyl

10. 4-( $\beta$-Phenylethyl)2-thiazolyl

11. 4- $\beta$-( $p$-Methoxyphenyl)ethyl-2-thiazolyl

12. 4-Phenyl-5-chloro2-thiazolyl

13. 4-(a-Naphthyl)-5chloro-2-thiazolyl

14. 4-( $\beta$-Naphthyl)-5chloro-2-thiazolyl

15. 4-(p-Hydroxyphenyl)5-chloro-2-thiazolyl

16. 4-(p-Methoxyphenyl)5-chloro-2-thiazolyl

17. 4-(p-Chlorophenyl)5-chloro-2-thiazolyl

18. 4-(p-Bromophenyl)5-chloro-2-thiazolyl

19. 4-( $\beta$-Phenylethyl)5-chloro-2-thiazolyl

20. 4- $\beta$ - ( $p$-Methoxyphenyl)ethyl-5-chloro2-thiazolyl

21. 4-Phenyl-5-bromo2-thiazolyl

22. 4-( $a$-Naphthyl)-5bromo-2-thiazolyl

23. 4-( $\beta$-Naphthyl)-5bromo-2-thiazolyl

24. 4-(p-Hydroxyphenyl)5-bromo-2-thiazolyl

\begin{tabular}{|c|c|c|c|c|c|c|c|c|}
\hline 124 & 72 & $\begin{array}{l}5.13 \\
\text { (Nitro }\end{array}$ & $\begin{array}{l}5.24 \\
\text { ogen) }\end{array}$ & - & - & 0 & 25 & $\begin{array}{l}\text { Carvularia } \\
\text { lunata }\end{array}$ \\
\hline 148 & 80 & 14.6 & 14.4 & - & - & 18 & 90 & "I \\
\hline 155 & 65 & - & - & 10.65 & 10.81 & 6 & 45 & $\begin{array}{l}\text { Piricularia } \\
\text { oryzae (Cav.) }\end{array}$ \\
\hline 120 & 68 & 一 & - & 8.92 & 9.25 & 4 & 50 & $"$ \\
\hline 95 & 62 & - & - & 8.88 & 9.25 & 4 & 50 & $"$ \\
\hline 180 & 65 & - & - & 10.02 & 10.25 & 7 & 52 & $"$ \\
\hline 175 & 62 & - & - & 9.48 & 9.81 & 4 & 50 & " \\
\hline 162 & 60 & - & - & 9.42 & 9.68 & $2 / 5^{*}$ & $25 / 45^{*}$ & $"$ \\
\hline 115 & 64 & - & - & 8.22 & 8.53 & $3 / 6^{*}$ & $30 / 55^{*}$ & " \\
\hline 187 & 72 & - & - & 9.44 & 9.88 & 4 & 45 & " \\
\hline 212 & 68 & - & - & 8.78 & 9.04 & 4 & 45 & " \\
\hline 110 & 62 & 10.12 & 10.74 & - & - & 3 & 30 & " \\
\hline 103 & 65 & 9.84 & 9.33 & - & - & 3 & 28 & " \\
\hline 186 & 65 & 9.76 & 9.33 & - & - & 3 & 32 & $"$ \\
\hline 181 & 61 & 10.52 & 10.24 & - & - & 5 & 40 & $"$ \\
\hline 173 & 65 & 10.12 & 9.85 & - & - & 5 & 40 & $"$ \\
\hline 169 & 62 & 19.64 & 19.45 & - & - & $3 / 6^{*}$ & $30 / 55^{*}$ & $" \prime$ \\
\hline 148 & 64 & 29.43 & 28.20 & - & 一 & $3 / 6^{*}$ & $30 / 58^{*}$ & $" 1$ \\
\hline 152 & 65 & 10.22 & 9.90 & - & - & 5 & 40 & " \\
\hline 130 & 65 & 9.82 & 9.14 & - & - & 4 & 35 & " \\
\hline 130 & 70 & 20.83 & 21.33 & - & - & 5 & 38 & $" 1$ \\
\hline 144 & 68 & 18.01 & 18.82 & - & - & 5 & 40 & " \\
\hline 158 & 65 & 17.98 & 18.82 & - & - & 4 & 75 & "I \\
\hline 207 & 58 & 19.63 & 20.46 & - & - & 5 & 40 & $" l$ \\
\hline
\end{tabular}


TABLE I. (Continued)

\begin{tabular}{|c|c|c|c|c|c|c|c|c|c|c|}
\hline \multirow[b]{2}{*}{$\begin{array}{l}\text { Sl. } \\
\text { No. }\end{array}$} & \multirow[b]{2}{*}{ Nature of R. } & \multirow[b]{2}{*}{$\mathrm{mp}_{\mathrm{C}}$} & \multirow[b]{2}{*}{$\underset{\%}{\text { Yield }}$} & \multicolumn{2}{|c|}{ Halogen $(\%)$} & \multicolumn{2}{|c|}{ Sulphur $(\%)$} & \multicolumn{3}{|c|}{ Fungicidal results } \\
\hline & & & & Found & Calc. & Found & Calc. & $\begin{array}{l}\text { Ger. at } \\
10 \text { ppm } \\
\%\end{array}$ & $\begin{array}{l}\text { Ger. at } \\
1 \text { ppm } \\
\%\end{array}$ & Test fungus \\
\hline 1 & 2 & 3 & 4 & 5 & 6 & 7 & 8 & 9 & 10 & 11 \\
\hline 25 . & $\begin{array}{l}\text { 4-(p-Methoxyphenyl)- } \\
\text { 5-bromo-2-thiazolyl }\end{array}$ & 115 & 60 & 18.65 & 19.75 & - & - & 4 & 36 & $\begin{array}{l}\text { Piricularia } \\
\text { oryzae (Cav.) }\end{array}$ \\
\hline 26. & $\begin{array}{l}\text { 4-(p-Chlorophenyl)- } \\
\text { 5-bromo-2-thiazolyl }\end{array}$ & 168 & 64 & 26.45 & 27.23 & - & - & $3 / 5^{*}$ & $31 / 45^{*}$ & " \\
\hline 27. & $\begin{array}{l}\text { 4-(p-Bromophenyl)- } \\
\text { 5-bromo-2-thiazolyl }\end{array}$ & 166 & 68 & 34.66 & 35.24 & - & - & $3 / 8^{*}$ & $30 / 70^{*}$ & " \\
\hline 28. & $\begin{array}{l}\text { 4-( } \beta \text {-Phenylethyl)- } \\
\text { 5-bromo-2-thiazolyl }\end{array}$ & 141 & 65 & 18.86 & 19.86 & - & - & 4 & 36 & $"$ \\
\hline 29. & $\begin{array}{l}\text { 4- } \beta \text {-( } p \text {-Methoxyphenyl)- } \\
\text { ethyl-5-bromo- } \\
\text { 2-thiazolyl }\end{array}$ & 155 & 62 & 17.62 & 18.48 & - & - & 3 & 30 & " \\
\hline 30. & $\begin{array}{l}\text { 4-Phenyl-5-iodo- } \\
\text { 2-thiazolyl }\end{array}$ & 108 & 52 & 29.12 & 30.09 & 一 & - & 3 & 35 & $" \prime$ \\
\hline 31. & $\begin{array}{l}\text { 4-(a-Naphthyl)- } \\
\text { 5-iodo-2-thiazolyl }\end{array}$ & 149 & 55 & 25.65 & 26.91 & - & - & 5 & 40 & $"$ \\
\hline 32. & $\begin{array}{l}\text { 4-( } \beta \text {-Naphthyl)-5- } \\
\text { iodo-2-thiazolyl }\end{array}$ & 123 & 55 & 25.51 & 26.91 & - & - & 4 & 34 & " \\
\hline 33. & $\begin{array}{l}\text { 4-( } p \text {-Hydroxylphenyl)- } \\
\text { 5-iodo-2-thiazolyl }\end{array}$ & 105 & 50 & 27.56 & 28.99 & - & - & 6 & 45 & $"$ \\
\hline 34. & $\begin{array}{l}\text { 4-(p-Methoxyphenyl)- } \\
\text { 5-iodo-2-thiazolyl }\end{array}$ & 100 & 52 & 27.11 & 28.09 & - & - & 6 & 47 & $"$ \\
\hline 35. & $\begin{array}{l}\text { 4-( } p \text {-Chlorophenyl)- } \\
\text { 5-iodo-2-thiazolyl }\end{array}$ & 118 & 54 & 34.72 & 35.58 & - & - & $3 / 8^{*}$ & $29 / 75^{*}$ & $"$ \\
\hline 36. & $\begin{array}{l}\text { 4-( } p \text {-Bromophenyl)- } \\
\text { 5-iodo-2-thiazolyl }\end{array}$ & 130 & 60 & 40.01 & 41.32 & - & - & $2 / 8^{*}$ & $25 / 78^{*}$ & " \\
\hline 37. & $\begin{array}{l}\text { 4-( } \beta \text {-Phenylethyl)- } \\
\text { 5-iodo-2-thiazoly] }\end{array}$ & 101 & 51 & 27.03 & 28.18 & - & - & 3 & 28 & " \\
\hline 38. & $\begin{array}{l}\text { 4- } \beta \text {-( } p \text {-Methoxyphenyl)- } \\
\text { ethyl-5-iodo- }\end{array}$ & 128 & 55 & 25.51 & 26.46 & - & - & 4 & 33 & $"$ \\
\hline
\end{tabular}
2-thiazolyl

\footnotetext{
39. Phenyl

40. (p)Tolyl

41. (p)Chlorophenyl

42. (p)Bromophenyl

43. (p)Iodophenyl

44. (p)Nitrophenyl

45. (a) Naphthyl

46. ( $\beta$ ) Naphthyl

47. 4-Phenyl-2-thiazolyl

48. 4-(a-Naphthyl)2-thiazolyl

49. 4-( $\beta$-Naphthyl)2-thiazolyl

50. 4-( $p$-Hydroxylphenyl)2-thiazolyl

51. 4-(p-Methoxyphenyl)2-thiazolyl
}

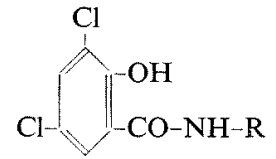

(Fungicidal assay in control-95\% germination)

\begin{tabular}{|c|c|c|c|c|c|c|c|c|}
\hline 112 & 64 & 24.64 & 25.18 & - & - & 10 & 70 & $"$ \\
\hline 161 & 65 & 23.48 & 23.99 & - & - & 11 & 70 & $"$ \\
\hline 175 & 70 & 33.14 & 33.65 & - & - & 10 & 70 & $"$ \\
\hline 182 & 70 & 41.62 & 41.83 & - & - & 11 & 75 & $"$ \\
\hline 127 & 68 & 47.82 & 48.53 & - & - & 12 & 78 & $"$ \\
\hline 142 & 65 & 21.43 & 21.72 & - & - & 12 & 75 & " \\
\hline 158 & 70 & 20.78 & 21.39 & 一 & - & 7 & 52 & $" \prime$ \\
\hline 168 & 72 & 20.81 & 21.39 & - & - & 10 & 68 & "r \\
\hline 210 & 64 & 19.05 & 19.45 & - & - & 4 & 34 & " \\
\hline 145 & 65 & 16.85 & 17.11 & - & - & 4 & 35 & "r \\
\hline 198 & 70 & 18.21 & 17.11 & - & - & 4 & 35 & " \\
\hline 222 & 68 & 19.02 & 18.66 & - & - & 3 & 29 & " \\
\hline 152 & 65 & 18.26 & 17.98 & - & - & 3 & 31 & " \\
\hline
\end{tabular}


Table I. (Continued)

\begin{tabular}{|c|c|c|c|c|c|c|c|c|c|c|}
\hline \multirow[b]{2}{*}{$\begin{array}{l}\text { Sl. } \\
\text { No. }\end{array}$} & \multirow[b]{2}{*}{ Nature of $R$. } & \multirow[b]{2}{*}{$\operatorname{mp}_{\mathrm{C}}$} & \multirow[b]{2}{*}{$\underset{\%}{\text { Yield }}$} & \multicolumn{2}{|c|}{ Halogen $(\%)$} & \multicolumn{2}{|c|}{ Sulphur $(\%)$} & \multicolumn{3}{|c|}{ Fungicidal results } \\
\hline & & & & Found & Calc. & Found & Calc. & $\begin{array}{l}\text { Ger, at } \\
10 \text { ppm } \\
\%\end{array}$ & $\begin{array}{l}\text { Ger, at } \\
1 \mathrm{ppm} \\
\%\end{array}$ & Test fungus \\
\hline 1 & 2 & 3 & 4 & 5 & 6 & 7 & 8 & 9 & 10 & 11 \\
\hline 52. & $\begin{array}{l}\text { 4-(p-Chlorophenyl)- } \\
\text { 2-thiazolyl }\end{array}$ & 134 & 66 & 27.21 & 26.66 & - & - & $5 / 7^{*}$ & $41 / 70^{*}$ & $\begin{array}{l}\text { Piricularia } \\
\text { oryzae }(\text { Cav.) }\end{array}$ \\
\hline 53. & $\begin{array}{l}\text { 4-( } p \text {-Bromophenyl)- } \\
\text { 2-thiazolyl }\end{array}$ & 147 & 55 & 32.88 & 34.01 & - & - & $5 / 7^{*}$ & $45 / 75^{*}$ & " \\
\hline 54. & $\begin{array}{l}\text { 4-( } \beta \text {-Phenylethyl)- } \\
\text { 2-thiazolyl }\end{array}$ & 184 & 64 & 18.35 & 18.07 & - & - & 4 & 35 & " \\
\hline 55. & $\begin{array}{l}\text { 4- } \beta \text {-( } p \text {-Methoxyphenyl)- } \\
\text { ethyl-2-thiazolyl }\end{array}$ & 138 & 60 & 17.12 & 16.79 & - & - & 4 & 30 & $"$ \\
\hline 56. & $\begin{array}{l}\text { 4-Phenyl-5-chloro- } \\
\text { 2-thiazolyl }\end{array}$ & 235 & 54 & 25.13 & 26.67 & - & - & 1 & 10 & $"$ \\
\hline 57. & $\begin{array}{l}\text { 4-(a-Naphthyl)-5- } \\
\text { chloro-2-thiazolyl }\end{array}$ & 138 & 60 & 22.62 & 23.70 & - & - & 1 & 10 & " \\
\hline 58. & $\begin{array}{l}\text { 4- }(\beta \text {-Naphthyl })-5- \\
\text { chloro-2-thiazolyl }\end{array}$ & 195 & 60 & 23.11 & 23.70 & - & - & 1 & 15 & $" 1$ \\
\hline 59. & $\begin{array}{l}\text { 4-(p-Hydroxyphenyl)- } \\
\text { 5-chloro-2-thiazolyl }\end{array}$ & 175 & 61 & 24.86 & 25.64 & - & - & 1 & 18 & " \\
\hline 60. & $\begin{array}{l}\text { 4-(p-Methoxyphenyl)- } \\
\text { 2-chloro-2-thiazolyl }\end{array}$ & 200 & 64 & 23.86 & 24.79 & - & - & 2 & 22 & " \\
\hline 61. & $\begin{array}{l}\text { 4-(p-Chlorophenyl)- } \\
\text { 5-chloro-2-thiazolyl }\end{array}$ & $230(d)$ & 62 & 31.83 & 32.72 & - & - & $0 / 0^{*}$ & $15 / 15^{*}$ & "r \\
\hline 62. & $\begin{array}{l}\text { 4-( } p \text {-Bromophenyl)- } \\
\text { 5-chloro-2-thiazolyl }\end{array}$ & 108 & 60 & 38.08 & 38.97 & - & - & $0 / 0^{*}$ & $15 / 18^{*}$ & " \\
\hline 63. & $\begin{array}{l}\text { 4-( } \beta \text {-Phenylethyl)- } \\
\text { 5-chloro-2-thiazolyl }\end{array}$ & 238 & 58 & 24.15 & 25.03 & - & - & 2 & 25 & $"$ \\
\hline 64. & $\begin{array}{l}\text { 4- } \beta \text {-( } p \text {-Methoxyphenyl)- } \\
\text { ethyl-5-chloro-2-thiazolyl }\end{array}$ & 156 & 60 & 22.82 & 23.28 & - & - & 3 & 30 & " \\
\hline 65. & $\begin{array}{l}\text { 4-( } p \text {-Phenyl)-5-bromo- } \\
\text { 2-thiazolyl }\end{array}$ & 225 & 62 & 33.18 & 34.01 & - & - & 2 & 24 & $"$ \\
\hline 66. & $\begin{array}{l}\text { 4-(a-Naphthyl)-5- } \\
\text { bromo-2-thiazolyl }\end{array}$ & 176 & 62 & 29.42 & 30.57 & - & - & 2 & 25 & $"$ \\
\hline 67. & $\begin{array}{l}4-(\beta \text {-Naphthyl })-5- \\
\text { bromo-2-thiazolyl }\end{array}$ & 162 & 68 & 29.51 & 30.57 & - & - & 3 & 30 & $"$ \\
\hline 68. & $\begin{array}{l}\text { 4-( } p \text {-Hydroxyphenyl)-5- } \\
\text { bromo-2-thiazolyl }\end{array}$ & 153 & 60 & 31.53 & 32.83 & - & - & 3 & 32 & " \\
\hline 69. & $\begin{array}{l}\text { 4-(p-Methoxyphenyl)-5- } \\
\text { bromo-2-thiazolyl }\end{array}$ & 112 & 58 & 30.62 & 31.85 & - & - & 3 & 32 & $"$ \\
\hline 70 & $\begin{array}{l}\text { 4-( } p \text {-Chlorophenyl)-5- } \\
\text { bromo-2-thiazolyl }\end{array}$ & 181 & 60 & 37.63 & 38.97 & - & - & $3 / 5^{*}$ & $30 / 50^{*}$ & $"$ \\
\hline 71. & $\begin{array}{l}\text { 4-( } p \text {-Bromophenyl)-5- } \\
\text { broms-2-thiazolyl }\end{array}$ & 198 & 60 & 4303 & 44.17 & - & - & $3 / 5^{*}$ & $30 / 45^{*}$ & "I \\
\hline 72. & $\begin{array}{l}\text { 4-( } \beta \text {-Phenylethyl })-5- \\
\text { bromo-2-thiazolyl }\end{array}$ & 160 & 62 & 31.21 & 32.00 & - & - & 3 & 32 & " \\
\hline 73. & $\begin{array}{l}\text { 4- } \beta \text { - }(p \text {-Methoxyphenyl)- } \\
\text { ethyl-5-bromo-2-thiazolyl }\end{array}$ & 112 & 60 & 29.31 & 30.08 & - & - & 4 & 36 & $"$ \\
\hline 74. & $\begin{array}{l}\text { 4-Phenyl-5-iodo- } \\
\text { 2-thiazolyl }\end{array}$ & 222 & 56 & 38.68 & 40.33 & - & - & 4 & 35 & $" \prime$ \\
\hline 75. & $\begin{array}{l}\text { 4-(a-Naphthyl)-5- } \\
\text { iodo-2-thiazolyl }\end{array}$ & 141 & 55 & 34.82 & 36.60 & - & - & 4 & 37 & $" 1$ \\
\hline 76. & $\begin{array}{l}\text { 4- }(\beta \text {-Naphthyl)-5- } \\
\text { iodo-2-thiazolyl }\end{array}$ & 217 & 56 & 35.01 & 36.60 & - & - & 4 & 37 & $"$ \\
\hline 77. & $\begin{array}{l}\text { 4-(p-Hydroxylphenyl)- } \\
\text { 5-iodo-2-thiazolyl }\end{array}$ & 146 & 55 & 37.82 & 39.05 & - & - & 3 & 35 & " \\
\hline 78. & $\begin{array}{l}\text { 4-( } p \text { methoxyphenyl)- } \\
\text { 5-iodo-2-thiazolyl }\end{array}$ & 196 & 56 & 36.55 & 38.01 & - & - & 3 & 32 & " \\
\hline
\end{tabular}


TABLE I. (Continued)

\begin{tabular}{|c|c|c|c|c|c|c|c|c|c|c|}
\hline \multirow[b]{2}{*}{$\begin{array}{l}\text { Sl. } \\
\text { No. }\end{array}$} & \multirow[b]{2}{*}{ Nature of $R$. } & \multirow[b]{2}{*}{${ }_{{ }^{\circ} \mathrm{Cp}}^{\mathrm{mp}}$} & \multirow[b]{2}{*}{$\underset{\%}{\text { Yield }}$} & \multicolumn{2}{|c|}{ Halogen $(\%)$} & \multicolumn{2}{|c|}{ Sulphur $(\%)$} & \multicolumn{3}{|c|}{ Fungicidal results } \\
\hline & & & & Found & Calc. & Found & Calc. & $\begin{array}{l}\text { Ger. at } \\
10 \text { ppm } \\
\%\end{array}$ & $\begin{array}{l}\text { Ger. at } \\
1 \mathrm{ppm} \\
\%\end{array}$ & Test fungus \\
\hline 1 & 2 & 3 & 4 & 5 & 6 & 7 & 8 & 9 & 10 & 11 \\
\hline 79. & $\begin{array}{l}\text { 4-(p-Chlorophenyl)- } \\
\text { 5-iodo-2-thiazolyl }\end{array}$ & 112 & 55 & 43.52 & 44.43 & - & - & $4 / 7^{*}$ & $50 / 60^{*}$ & $\begin{array}{l}\text { Piricularia } \\
\text { oryzae (Cav.) }\end{array}$ \\
\hline 80. & $\begin{array}{l}\text { 4-(p-Bromophenyl)- } \\
\text { 5-iodo-2-thiazolyl }\end{array}$ & 200 & 54 & 47.13 & 48.76 & - & - & $5 / 7^{*}$ & $52 / 65^{*}$ & " \\
\hline 81. & $\begin{array}{l}\text { 4-( } \beta \text {-Phenylethyl)- } \\
\text { 5-iodo-2-thiazolyl }\end{array}$ & 216 & 52 & 37.21 & 38.15 & - & - & 4 & 45 & " \\
\hline 82. & $\begin{array}{l}\text { 4- } \beta \text {-( } p \text {-Methoxyphenyl)- } \\
\text { ethyl-5-iodo-2-thiazolyl }\end{array}$ & 186 & 54 & 35.12 & 36.07 & - & - & 4 & 42 & $"$ \\
\hline
\end{tabular}

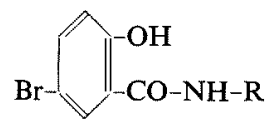
83. (o)Bromophenyl
84. ( $p$ )Bromophenyl
85. (o)Chlorophenyl
86. ( $p$ )Chlorophenyl
87. ( $\beta)$ Naphthyl
88. 4-( $\beta$-Phenylethyl)- 2-thiazolyl
89. 4-Phenyl-5-bromo- 2-thiazolyl
90. 4-( $\beta$-Phenylethyl)- 5-bromo-2-thiazolyl
91. 4-(p-Tolyl)-5- bromo-2-thiazolyl

(Fungicidal assay in control-94\% germination)

$\begin{array}{ccccccccc}152 & 63 & 43.35 & 43.12 & - & - & 0 & 20 & \begin{array}{c}\text { Carvularia } \\ \text { lunata }\end{array} \\ 176 & 72 & 42.60 & 43.12 & - & - & 0 & 20 & " \\ 171 & 82 & 35.03 & 35.37 & - & - & 10 & 75 & " \\ 210 \sim 212 & 60 & 35.20 & 35.37 & - & - & 4 & 50 & " \\ 176 & 68 & 23.83 & 23.40 & - & - & 6 & 50 & " \\ 84 & 55 & 20.06 & 20.00 & - & - & 23 & 90 & " \\ 130 & 60 & 33.53 & 35.26 & - & - & 27 & 90 & " \\ 104 & 60 & 33.53 & 33.33 & - & - & 1 & 20 & " \\ 98 & 58 & 34.08 & 34.18 & - & - & 32 & 90 & "\end{array}$

92. Phenyl

(Fungicidal assay in control-97\% germination)

93. (o)Chlorophenyl

\begin{tabular}{|c|c|c|c|c|c|c|c|c|}
\hline $155 \sim 56$ & 80 & $\begin{array}{l}4.87 \\
\text { (Nitrc }\end{array}$ & $\begin{array}{l}5.32 \\
\text { gen) }\end{array}$ & - & - & 17 & 90 & $\begin{array}{l}\text { Piricularia } \\
\text { oryzae (Cav.) }\end{array}$ \\
\hline 140 & 72 & 12.21 & 11.94 & - & - & 18 & 95 & " \\
\hline 198 & 65 & 11.62 & 11.94 & - & - & 15 & 85 & $"$ \\
\hline 175 & 70 & 11.64 & 11.94 & - & 一 & 12 & 90 & " \\
\hline 188 & 68 & 22.92 & 23.24 & - & - & 13 & 90 & $"$ \\
\hline $199 \sim 200$ & 65 & - & - & 9.62 & 9.25 & 16 & 90 & $"$ \\
\hline $150 \sim 151$ & 70 & - & - & 7.32 & 7.53 & 16 & 90 & $"$ \\
\hline 183 & 68 & - & - & 8.94 & 8.82 & 15 & 90 & $"$ \\
\hline 215 & 68 & - & - & 9.63 & 9.41 & 13 & 90 & $"$ \\
\hline 186 & 65 & - & - & 8.12 & 8.54 & 10 & 89 & $"$ \\
\hline 182 & 64 & - & - & 8.93 & 8.43 & 12 & 90 & $"$ \\
\hline 178 & 64 & - & - & 9.37 & 9.76 & 10 & 90 & $"$ \\
\hline
\end{tabular}

4. (m)Chlorophenyl

95. (p)Chlorophenyl

96. (p)Bromophenyl

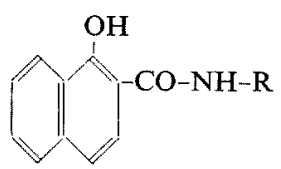

97. 4-Phenyl-2-thiazolyl

98. 4-(p-Bromophenyl)2-thiazolyl

99. 4-( $\beta$-Phenylethyl)2-thiazolyl

100. 4-(Isobutyl)-2-thiazolyl 101. 4-(p-Methoxyphenyl)-
2-thiazolyl

102. 4-( $p$-Chlorophenyl)2-thiazolyl

103. 4-Methyl-5-carbethoxy2-thiazolyl 
TABle I. (Continued)

\begin{tabular}{|c|c|c|c|c|c|c|c|c|c|c|}
\hline \multirow[b]{2}{*}{$\begin{array}{l}\text { Sl. } \\
\text { No. }\end{array}$} & \multirow[b]{2}{*}{ Nature of $R$. } & \multirow[b]{2}{*}{$\operatorname{mp}_{{ }^{\circ} \mathrm{C}}$} & \multirow[b]{2}{*}{$\underset{\%}{\text { Yield }}$} & \multicolumn{2}{|c|}{ Halogen $(\%)$} & \multicolumn{2}{|c|}{ Sulphur $(\%)$} & \multicolumn{3}{|c|}{ Fungicidal results } \\
\hline & & & & Found & Calc. & Found & Calc. & $\begin{array}{l}\text { Ger. at } \\
10 \text { ppm } \\
0\end{array}$ & $\begin{array}{l}\text { Ger. at } \\
1 \text { ppm } \\
\%\end{array}$ & Test fungus \\
\hline 1 & 2 & 3 & 4 & 5 & 6 & 7 & 8 & 9 & 10 & 11 \\
\hline 04. & $\begin{array}{l}\text { 4-Phenyl-5-bromo- } \\
\text { 2-thiazolyl }\end{array}$ & 169 & 66 & - & - & 6.98 & 7.53 & 11 & 80 & $\begin{array}{l}\text { Piricularia } \\
\text { oryzae (Cav.) }\end{array}$ \\
\hline 05 & $\begin{array}{l}\text { 4-(p-Bromophenyl)- } \\
\text { 5-bromo-2-thiazolyl }\end{array}$ & 180 & 70 & 一 & - & 6.10 & 6.35 & 3 & 30 & " \\
\hline 06 & $\begin{array}{l}\text { 4-( } p \text {-Methoxyphenyl)- } \\
\text { 5-bromo-2-thiazolyl }\end{array}$ & 168 & 71 & - & - & 6.84 & 7.03 & 6 & 52 & $"$ \\
\hline 107. & $\begin{array}{l}\text { 4-( } p \text {-Chlorophenyl)- } \\
\text { 5-bromo-2-thiazolyl }\end{array}$ & 250 & 72 & - & - & 6.63 & 6.96 & 3 & 31 & $"$ \\
\hline
\end{tabular}

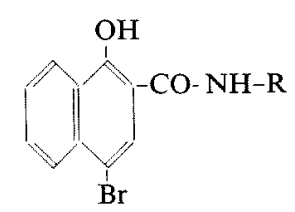

(Fungicidal assay in control-95\% germination)

108. Phenyl

109. (o)Chlorophenyl

110. (m)Chlorophenyl

111. (p)Chlorophenyl

112. (p)Bromophenyl

113. 4-Phenyl-2-thiazolyl

114. 4-(p-Bromophenyl)2-thiazolyl

115. 4-( $\beta$-Phenylethyl)2-thiazolyl

116. 4-(Isobutyl)-2-thiazolyl $>250$

117. 4-(p-Methoxyphenyl)- 185 2-thiazolyl

118. 4-(p-Chlorophenyl)2-thiazolyl

119. 4-Methyl-5-carbethoxy2-thiazolyl

120. 4-Phenyl-5-bromo2-thiazolyl

121. 4-( $p$-Bromophenyl)5-bromo-2-thiazolyl

122. 4-(p-Methoxyphenyl)5-bromo-2-thiazolyl

123. 4-( $p$-Chlorophenyl)5-bromo-2-thiazolyl

$$
\begin{array}{llll}
192 & 68 & 23.20 & 23.40
\end{array}
$$$$
\begin{array}{llllll}
180 & 72 & 30.45 & 30.67 & -\quad & -
\end{array}
$$$$
\begin{array}{llllllll}
218 & 68 & 30.61 & 30.67 & - & - & 9 & 70
\end{array}
$$$$
\begin{array}{lllllll}
195 & 66 & 30.20 & 30.67 & - & - & 9
\end{array}
$$$$
\begin{array}{lllllll}
215 & 60 & 37.73 & 37.99 \quad-\quad & - & 12 & 85
\end{array}
$$$$
\begin{array}{llllllll}
195 & 70 & 18.57 & 18.82 & - & - & 5 & 40
\end{array}
$$$$
\begin{array}{llllllll}
195 \quad 71 \quad 31.54 & 31.74 \quad & - & 13 & 85
\end{array}
$$$$
\begin{array}{llllllll}
182 & 72 & 17.45 & 17.66 & - & - & 14 & 19
\end{array}
$$$$
\begin{array}{lllllll}
250 \quad 70 & 19.48 & 19.75 & - & - & 12 & 81
\end{array}
$$$$
\begin{array}{lll}
190 & &
\end{array}
$$$$
\begin{array}{llllll}
190 & 68 & 24.91 & 25.14 & - & -
\end{array}
$$$$
\begin{array}{lllllllll}
212 & 68 & 31.48 & 31.74 & - & - & 3 & 8
\end{array}
$$$$
>250 \quad 68 \quad 40.75 \quad 41.09 \quad \text { - } \quad \text { - } 755
$$$$
231 \quad 70 \quad 29.64 \quad 29.90 \quad-\quad-\quad 10 \quad 1080
$$$$
\begin{array}{lllllll}
192 \quad 70 & 35.98 & 36.24 & - & & & 5
\end{array} 40
$$<smiles>[R]NC(=O)c1cc(O)c2ccccc2c1</smiles>

(Fungicidal assay in control-95\% germination)

$\begin{array}{lllllll}243 & 70 & \begin{array}{l}4.94 \quad 5.32 \\ \text { (Nitrogen) }\end{array} & & - & 17 & 95 \\ 221 & 71 & \begin{array}{l}4.58 \quad 5.04 \\ \text { (Nitrogen) }\end{array} & & - & 15 & 90 \\ 222 & 65 & \begin{array}{l}4.12 \quad 4.47 \\ \text { (Nitrogen) }\end{array} & & - & 12 & 85\end{array}$


TABle I. (Continued)

\begin{tabular}{|c|c|c|c|c|c|c|c|c|c|c|}
\hline \multirow[b]{2}{*}{$\begin{array}{l}\text { Sl. } \\
\text { No. }\end{array}$} & \multirow[b]{2}{*}{ Nature of $\mathrm{R}$. } & \multirow[b]{2}{*}{$\underset{{ }^{\mathrm{mp}} \mathrm{C}}{\mathrm{mp}}$} & \multirow[b]{2}{*}{$\underset{\%}{\text { Yield }}$} & \multicolumn{2}{|c|}{ Halogen $(\%)$} & \multicolumn{2}{|c|}{ Sulphur $(\%)$} & \multicolumn{3}{|c|}{ Fungicidal results } \\
\hline & & & & Found & Calc. & Found & Calc. & $\begin{array}{l}\text { Ger. at } \\
10 \text { ppm } \\
\text { \% }\end{array}$ & $\begin{array}{l}\text { Ger. at } \\
1 \text { ppm } \\
\%\end{array}$ & Test fungus \\
\hline 1 & 2 & 3 & 4 & 5 & 6 & 7 & 8 & 9 & 10 & 11 \\
\hline 127. & $(\beta)$ Napthyl & 243 & 66 & $\begin{array}{l}3.98 \\
\text { (Nitro }\end{array}$ & $\begin{array}{l}4.47 \\
\text { gen) }\end{array}$ & - & - & 10 & 80 & $\begin{array}{l}\text { Piricularia } \\
\text { oryzae (Cav.) }\end{array}$ \\
\hline 128. & (o)Chlorophenyl & 225 & 66 & 11.78 & 11.94 & - & - & 16 & 90 & " \\
\hline 129. & (m)Chlorophenyl & 241 & 68 & 11.69 & 11.94 & - & - & 15 & 85 & " \\
\hline 130. & $(p)$ Chlorophenyl & 238 & 70 & 11.81 & 11.94 & - & - & 12 & 80 & $"$ \\
\hline 131. & (p)Bromophenyl & $248 \sim 49$ & 70 & 22.92 & 23.24 & - & - & 13 & 85 & $" r$ \\
\hline 132. & 4-Phenyl-2-thiazolyl & $>250$ & 65 & - & - & 8.87 & 9.25 & 14 & 85 & $"$ \\
\hline 133. & $\begin{array}{l}\text { 4-( } p \text {-Bromophenyl)- } \\
\text { 2-thiazolyl }\end{array}$ & 233 & 64 & - & - & 7.38 & 7.53 & 4 & 45 & $" r$ \\
\hline 134. & $\begin{array}{l}\text { 4-(p-Chlorophenyl)- } \\
\text { 2-thiazolyl }\end{array}$ & 250 & 70 & - & - & 8.12 & 8.43 & 6 & 55 & $"$ \\
\hline 135. & $\begin{array}{l}\text { 4-(p-Methoxyphenyl)- } \\
\text { 2-thiazolyl }\end{array}$ & 195 & 72 & - & - & 8.32 & 8.54 & 8 & 60 & $"$ \\
\hline 136. & $\begin{array}{l}\text { 4-Phenyl-5-bromo- } \\
\text { 2-thiazolyl }\end{array}$ & 130 & 68 & - & - & 7.21 & 7.53 & 5 & 50 & $"$ \\
\hline 137. & $\begin{array}{l}\text { 4-( } p \text {-Bromophenyl })-5 \text { - } \\
\text { bromo-2-thiazolyl }\end{array}$ & $>250$ & 68 & - & - & 5.98 & 6.35 & 7 & 50 & " \\
\hline 138. & $\begin{array}{l}4 \text { - }(p \text {-Chlorophenyl)-5- } \\
\text { bromo-2-thiazolyl }\end{array}$ & 175 & 66 & - & - & 6.61 & 6.96 & 5 & 45 & " \\
\hline 139. & $\begin{array}{l}\text { 4-(p-Methoxyphenyl)- } \\
\text { 5-bromo-2-thiazolyl }\end{array}$ & 200 & 66 & - & - & 6.82 & 7.03 & 7 & 50 & " \\
\hline
\end{tabular}

140. Phenyl

141. (p)Tolyl

142. (a) Naphthyl

143. ( $\beta$ ) Naphthyl

144. (o)Chlorophenyl

145. ( $m$ ) Chlorophenyl

146. (p)Chlorophenyl

147. ( $p$ )Bromophenyl

148. 4-Phenyl-2-thiazolyl

149. 4-(p-Bromophenyl)2-thiazolyl

150. 4-( $p$-Chlorophenyl)2-thiazolyl

151. 4-(p-Methoxyphenyl)2-thiazolyl

152. 4-Phenyl-5-bromo2-thiazolyl

153. 4-( $p$-Bromophenyl)5-bromo-2-thiazolyl

154. 4-( $p$-Chlorophenyl)5-bromo-2-thiazolyl

155. 4-(p-Methoxyphenyl)5-bromo-2-thiazolyl

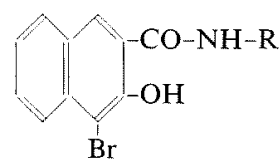

(Fungicidal assay in control-96\% germination)

\begin{tabular}{|c|c|c|c|c|c|c|c|c|}
\hline 161 & 70 & 23.24 & 23.40 & - & - & 9 & 70 & " \\
\hline 105 & 71 & 22.10 & 22.44 & - & - & 9 & 75 & $" \prime$ \\
\hline 152 & 70 & 20.21 & 20.40 & - & - & 9 & 72 & " \\
\hline 135 & 66 & 20.74 & 20.40 & - & - & 9 & 70 & " \\
\hline 203 & 64 & 30.68 & 30.67 & - & - & 10 & 75 & "I \\
\hline 160 & 65 & 31.21 & 30.67 & - & - & 9 & 70 & $"$ \\
\hline 215 & 70 & 30.68 & 30.67 & - & - & 9 & 75 & " \\
\hline 190 & 68 & 38.31 & 38.00 & - & - & 12 & 80 & " \\
\hline 250 & 68 & 18.57 & 18.82 & - & - & 12 & 80 & " \\
\hline 210 & 70 & 31.54 & 31.74 & - & - & 4 & 28 & $"$ \\
\hline 200 & 71 & 24.91 & 25.14 & - & - & 5 & 31 & $"$ \\
\hline 225 & 70 & 17.32 & 17.58 & - & - & 7 & 50 & "I \\
\hline 220 & 68 & 31.48 & 31.74 & - & - & 4 & 30 & " \\
\hline 238 & 71 & 40.75 & 41.09 & - & - & 6 & 45 & $"$ \\
\hline 220 & 70 & 35.98 & 36.24 & - & - & 3 & 30 & "I \\
\hline 232 & 70 & 29.64 & 29.90 & - & - & 5 & 40 & " \\
\hline
\end{tabular}


Acknowledgement. The authors are thankful to the Board of Scientific and Industrial Research, Government of Orissa for a research grant and to Dr. S. Y. Padmanavan, D. Sc., Director of the Central Rice Research Institute, Cuttack for granting permission to carry out the fungicidal study in his laboratory under his guidance.

\section{REFERENCES}

1) M. M. Rotmistov, G. V. Kulik, N. D. Mikknovskaya, I. I. Potostoskii and R. Zakor- nienko, U.S. Pat., 158, 156; Oct. 18, 1963.

2) S. Leonhard, Ger. Pat., 945, 446; Jul. 12, 1956.

3) M. R. Baichwal, L. G. Shah and S. G. Despande, Indian J. Pharm., 27, 2858 (1965).

4) B. C. Dash and G. N. Mahapatra, J. Instn. Chem. India, 39, 178 (1967).

5) G. N. Mahapatra, J. Indian Chem. Soc., 33, 527 (1956).

6) Y. Garreau, Compt. rend., 232, 847 (1951).

7) H. B. S. Montgomery and M. H. Moore, $J$. Pomol. and Hort. Sci., 15, 253 (1938). 